\title{
US deploys rapid response teams to hospitals with Ebola cases
}

\author{
Michael McCarthy
}

Seattle

The US government will deploy rapid response teams to local hospitals to help them handle any new Ebola cases, the Centers for Disease Control and Prevention (CDC) announced on Tuesday 14 October.

Tom Frieden, CDC director, said, "For any hospital anywhere in the country that has a confirmed case of Ebola, we will put a team on the ground within hours with some of the world's leading experts in how to take care of and protect healthcare workers from Ebola infection." The teams will include experts in infection control, laboratory science, management of Ebola treatment units, and administration of experimental treatments, he said.

The CDC is taking the action after a young female nurse became infected through taking care of a Liberian man with Ebola at a hospital in Dallas, Texas. ${ }^{1}$ Her infection prompted questions as to whether US hospitals are adequately prepared to handle Ebola patients.

The patient, Thomas Eric Duncan, 42, had been exposed to an Ebola patient in Liberia but did not develop symptoms until several days after he arrived in Dallas. He first sought care on 25 September at Texas Health Presbyterian Hospital, where he complained of fever, abdominal pain, and decreased urine output. While in the emergency department his temperature reached $103^{\circ} \mathrm{F}\left(39.4^{\circ} \mathrm{C}\right)$.

Although he had told the emergency room staff that he had recently been in west Africa, a diagnosis of Ebola infection was not made, and he was discharged on antibiotics with a diagnosis of sinusitis. Duncan returned to the hospital by ambulance on 28 September, and Ebola was subsequently diagnosed. Despite intensive care, which included ventilator support, renal dialysis, and treatment with an experimental antiviral drug, Duncan died on 8 October. $^{2}$

The nurse, 26 year old Nina Pham of Fort Worth, Texas, who had extensive contact with Duncan during his hospitalization, was admitted to the hospital two days later on 10 October, after she developed a low grade fever. The diagnosis of Ebola infection was confirmed over the weekend. She remains in good condition in an isolation unit at the Dallas hospital. Investigators have not yet determined how Pham became infected, and 76 of the hospital staff who were in contact with Duncan - or with materials such as blood samples that might have been contaminated-are now being monitored for signs and symptoms of infection.
A number of nurses' organizations have complained that their members have not been properly trained to care for Ebola patients. In an informal survey of 2000 members of National Nurses United, the largest US nurses' organization, 76\% said that their hospital had not communicated to them any policy on admitting patients infected with Ebola, and more than a third said that their hospital had insufficient supplies of face shields and impermeable gowns.

RoseAnn DeMoro, executive director of National Nurses United, said that nurses should be given hazmat (hazardous material) suits and improved training in the use of protective gear. "There is no standard short of optimal in protective equipment and hands-on training that is acceptable," DeMoro said.

Frieden said that, although the CDC had deployed infection control experts to the Dallas hospital, "in retrospect with 20/20 hindsight we could have sent a more robust inspection control team and been more hands-on with the hospital from day one." He added, "I've been hearing loud and clear from healthcare workers from around the country that they are worried, that they don't feel prepared to take care of a patient with Ebola."

Under the new protocols being put in place at the Dallas hospital an infection control manager will be onsite 24 hours a day, hospital staff will undergo additional training led by two nurses from the high level biocontainment unit at Emory University Hospital in Atlanta, Georgia, and the number of staff allowed to enter the hospital's isolation unit will be kept to a minimum. Asked whether future Ebola cases might be transferred to Emory or one of the three other high level biocontainment units in the United States, Frieden said that the option was being considered.

Texas health officials have identified 48 people in Dallas who were, or possibly were, in contact with Duncan while he was contagious. Those people have now been without symptoms for 14 or more days - two thirds of the virus's 21 day incubation period-since their last contact with Duncan.

"While it wouldn't be impossible that some of them would develop the disease," Frieden said, "they have now passed through the highest risk period, and it's decreasingly likely that any of them will develop Ebola."

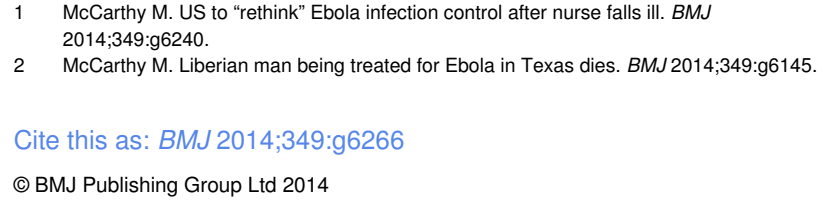


